\title{
Etude de l'implantation épiphylle de Xanthomo- nas campestris pv. corylina, $X$. campestris pv. juglandis, Erwinia herbicola et Pseudomonas paucimobilis sur feuilles de noisetier
}

\author{
Olivier PRUVOST \& Louis GARDAN \\ avec la collaboration technique de Martine Devaux \\ I.N.R.A., Station de Pathologie végétale et Phytohactiriologie, Route de Saint-Clément, Beaucouzí - F 49000 \\ Angers
}

Mots clés additionnels : Vie épiphỵte, microscopie ćlectromique à balay'age herbicola and Pseudomonas paucimobilis on hazelmut leaves.

Experimental study of the epiphyllous survival of a pathogenic homologous bactcrium $(X . c . p v$. cor $y$ lina $)$, of a pathogenic heterologous bacterium ( $X . C$. pv. juglandis) and of two saprophytic bacteria ( $E$. herhicola et $P$. paucimohilis) on hazelnut showed that only $X$. c. pv cor!lina multiplied intensively and stayed between $10^{6}$ and $10^{7}$ c.f.u./leaf. The other bacteria only reached $10^{3}$ to $10^{5}$ c.f.u./leaf. Observations with a seanning electron microscope showed that the bacteria first spread at random over the leaf and were then located in the hollows, on veins and sometimes on the lamina. The xanthomonads agglutinated in a mucus, which later cracks.

Additionnal key words : Scanning electron microcopes.

\section{INTRODUCTION}

Les organes aériens des plantes, en particulier les feuilles, sont colonisés par des microflores variées composées de bactéries, champignons, levures etc...

De nombreuses bactéries phytopathogènes appartenant aux genres Pseudomonas et Xanthomonas ont une phase épiphylle résidente au sens de LEBEN (1965); ces bactéries jouent un rôle important dans les cycles biologiques des maladies comme source potentielle d'inoculum (CRosse, 1963). C'est ainsi que X. campestris pv. corvlina est présent sur feuille de noisetier $\left(10^{4}\right.$ à $10^{8}$ c.f.u./feuille) tant en vergers qu'en pépinières malades, pendant toute la période végétative (GARDAN \& DeVAuX, 1984). La vie épiphylle d'autres Xanthomonas a été prouvée: par exemple, $X$. campestris pv. pruni (DHANVANTARI, 1973) et $X$. campestris pv. manihotis (DANIEL \& BOHER, 1978).

Les sites de survie des bactéries épiphylles sont principalement les dépressions existant entre les cellules épidermiques des feuilles et des nervures, les stomates, les hydathodes et les trichomes ou leur voisinage immé- 
diat; ils peuvent être occupés aussi bien par les organismes saprophytes que pathogènes. La face inférieure des feuilles semble plus favorable à la survie bactérienne que la face supérieure (LEBEN \& DAFT, 1964 ; LEBEN, 1969 ; LEBEN, 1974 ; MILES et al., 1977 ; SCHNEIDER \& GROGAN, 1977 ; BASHAN et al., 1981 ; GITAITIS et al., 1981 ; RoOs \& HATTINGH, 1983 ; Mew et al., 1984).

Nous avons abordé l'étude de certains aspects de la phase épiphylle sous un angle expérimental en comparant, sur feuilles de noisetier, l'implantation, la survie quantitative et la répartition topographique vue au microscope électronique à balayage d'une bactérie pathogène homologue : X.c. pv. corylina, d'une bactérie pathogène hétérologue : $X . c$. pv. juglandis et de deux bactéries pathogènes saprophytes: $E$. herbicola et $P$. paucimobilis.

\section{MATÉRIEL ET MÉTHODES}

\section{A. Inoculation des arbres}

Des marcottes d'un an de la variété «Fertile de Coutard » ont été plantées en pot et gardées à l'extérieur, à l'abri des précipitations.

Toutes les feuilles de certaines pousses herbacées ont été inoculées par trempage individuel pendant quelques secondes dans une suspension bactérienne titrée de l'une des quatre souches mentionnées dans le tableau 1. Sur chaque plant, des pousses « témoin » ont été trempées dans l'eau stérile. Le premier prélèvement est effectué juste après ressuyage des feuilles, les suivants sont faits $1,2,5,8,15,25,48$ et 82 jours après l'inoculation.

\section{B. Comptage des bactéries}

Au milieu LPGA (extrait de levure $7 \mathrm{~g}$, bactopeptone $7 \mathrm{~g}$, glucose $7 \mathrm{~g}$, agar $15 \mathrm{~g}$, eau distillée q.s.p. $1000 \mathrm{ml}$, pH 7,2) sont additionnés :

- de la cycloheximide $(50 \mathrm{mg} / \mathrm{l})$ pour tous les comptages,

- de la streptomycine $(25 \mathrm{mg} / 1)$ pour les comptages de $P$. paucimobilis et $X$. c. pv. corylina,
- de la rifamycine ( $25 \mathrm{mg} / \mathrm{l})$ pour les comptages d' $E$. herbicola,

- de la kanamycine $(10 \mathrm{mg} / 1)$ pour X.c. pv. juglandis.

Cinq feuilles, prélevées au hasard, sont lavées dans un récipient contenant $50 \mathrm{ml}$ d'eau stérile, par une agitation vigoureuse pendant une minute. Un prélèvement de 0,5 $\mathrm{ml}$ d'eau de lavage est utilisé pour un premier dénombrement des bactéries. Les mêmes feuilles sont ensuite broyées dans l'eau de lavage dans un bol en inox de $200 \mathrm{ml}$ à l'aide d'un broyeur type Waring-Blendor.

Les dénombrements des bactéries sont effectués sur milieu gélosé par dépôt de gouttes calibrées $(0,05 \mathrm{ml})$ des liquides de broyage et de lavage et leurs dilutions au $1 / 10$.

\section{Préparation des échantillons pour l'observation au microscope électronique à balayage}

A chaque comptage de bactéries, des portions de limbe sont prélevées au niveau de la nervure principale au $2 / 3 \mathrm{du}$ sommet, et en bordure en position médiane de la feuille. Les traitements suivants leur sont appliqués:

- fixation dans une solution de glutaraldéhyde à 4 p. 100 obtenue à partir d'une solution à 25 p. 100 diluée dans une solution tampon $\mathrm{pH} 7,2\left(\mathrm{KH}_{2} \mathrm{PO}_{4}\right.$ : $2,723 \mathrm{~g}, \mathrm{Na}_{2} \mathrm{HPO}_{4}\left(12 \mathrm{H}_{2} \mathrm{O}\right): 16,713 \mathrm{~g}$, eau ultrapure q.s.p. $1000 \mathrm{ml}$ ) pendant $90 \mathrm{mn}$ à $4{ }^{\circ} \mathrm{C}$ sous vide,

- immersion dans la solution tampon pH 7,2 pendant $12 \mathrm{~h}$ ou plus à $4{ }^{\circ} \mathrm{C}$,

- rinçage de $10 \mathrm{mn}$ dans trois bains de la solution tampon $\mathrm{pH} 7,2$ puis dans trois autres bains d'eau distillée,

- déshydratation dans l'éthanol à $70^{\circ}$ pendant $20 \mathrm{mn}$, puis dans l'éthanol à $95^{\circ}$ pendant $20 \mathrm{mn}$, puis dans l'éthanol absolu (trois bains de $20 \mathrm{mn}$ ),

- passage dans trois bains d'acétone pendant $10 \mathrm{mn}$,

- dessiccation selon la technique du point critique (BALZERS UNION CPD 010),

- métallisation à l'or par ionisation sous vide (appareil EMSCOPE AE 1230, $7 \mathrm{MA}, 1,2 \mathrm{kv}, 3 \mathrm{mn}$ ).

TABLEAU 1

Caractéristiques des quatre souches bactériennes implantées sur feuilles de Noisetier.

Characteristics of the four bacterial strains settled on hazelnut leaves.

\begin{tabular}{|c|c|c|c|c|c|}
\hline $\begin{array}{l}\text { Bactéries } \\
\text { implantées }\end{array}$ & $\begin{array}{l}\text { Numéro } \\
\text { de souche }\end{array}$ & $\begin{array}{l}\text { Caractères de } \\
\text { résistance }\end{array}$ & $\begin{array}{c}\text { Date } \\
\text { d'implantation }\end{array}$ & $\begin{array}{c}\text { Titre de } \\
\text { suspension } \\
\text { (c.f.u./ml) }\end{array}$ & $\begin{array}{c}\text { Hôte } \\
\text { d'origine }\end{array}$ \\
\hline X.c. pv. corvlina & $\mathrm{K} 100.1 \mathrm{Sm}^{\mathrm{R}}$ & Streptomycine & $23 / 05 / 85$ & $2,4 \cdot 10^{8}$ & Noisetier \\
\hline X.c. pv. juglandis & $1022 \mathrm{~K}^{\mathrm{R}}$ & Kanamycine & $23 / 05 / 85$ & $7,6 \cdot 10^{8}$ & Noyer \\
\hline E. herbicola & P 19.9 & $\begin{array}{l}\text { Rifamycine } \\
\text { (résistance } \\
\text { naturelle) }\end{array}$ & $24 / 06 / 85$ & $4,4 \cdot 10^{8}$ & Noisetier \\
\hline P. paucimobilis & $\mathrm{T} 43.3$ & $\begin{array}{l}\text { Streptomycine } \\
\text { (résistance } \\
\text { naturelle) }\end{array}$ & $24 / 06 / 87$ & $6,0 \cdot 10^{8}$ & Noisetier \\
\hline
\end{tabular}




\section{RÉSULTATS}

\section{A. Dynamique de l'installation et de la survie des bacté- ries}

L'implantation épiphylle par trempage des feuilles dans les quatre différentes suspensions bactériennes a permis de suivre séparément l'évolution quantitative du nombre moyen de bactéries par feuille.

Le lavage des bactéries permet de compter les bactéries installées superficiellement sur le relief de la feuille ou faiblement liées aux cellules épidermiques. Le broyage, par contre, donne la population totale y compris interne.

Pour X.c. pv. corylina le nombre total moyen de bactéries par feuille (fig. 1) est :

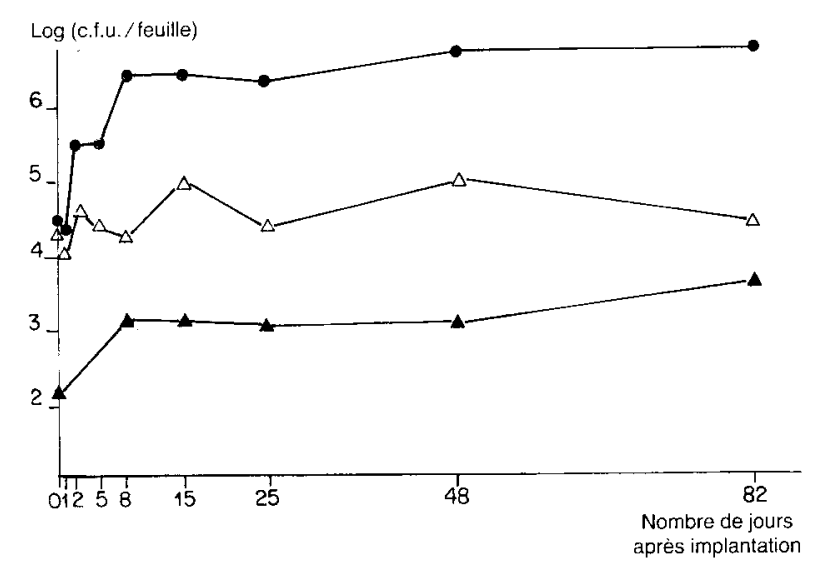

Figure 1

Evolution d'une population de Xanthomonas campestris pv. corylina implantée sur feuilles de noisetier: $\triangle$ comptage après lavage, comptage après lavage et broyage, $\boldsymbol{\Delta}$ flore naturelle des témoins.

Development of a Xanthomonas campestris $p v$. corylina population settled on hazelnut leat's: $\triangle$ counting after washing, $\bullet$ counting after washing and blending, $\mathbf{\Lambda}$ natural flora of control samples.

- d'environ $10^{4}$ juste après la contamination,

- diminue légèrement pendant le $\mathbf{l}^{\mathrm{cr}}$ jour,

- atteint $2,510^{6}$ au bout de 8 jours car les bactéries se sont multipliées,

- se maintient entre 1 et $710^{6}$ jusqu'à 82 jours.

Une analyse de variance montre une différence significative à $1 \mathrm{p}$. 100 entre les résultats obtenus par les deux techniques mais ne montre pas de différence significative de chacune des populations obtenues aux différentes dates d'analyse.

La comparaison des valeurs obtenues pour chaque date par les deux techniques, à l'aide de la p.p.d.s. donne des différences significatives à 8 et 82 jours et presque significatives à 25 jours. Entre 0 et 5 jours aucune différence significative n'est observée. L'utilisation du coefficient de Spearman montre que la différence entre le nombre de bactéries obtenues par lavage et le nombre total de bactéries augmente au cours du temps.

Il y a en moyenne 100 à 1000 fois plus de $X$. c. pv. corylina que de bactéries saprophytes naturellement présentes sur les feuilles.

L'évolution du nombre de $X . c$. pv. juglandis est indiqué sur la fig. 2 ; les courbes sont comparables à

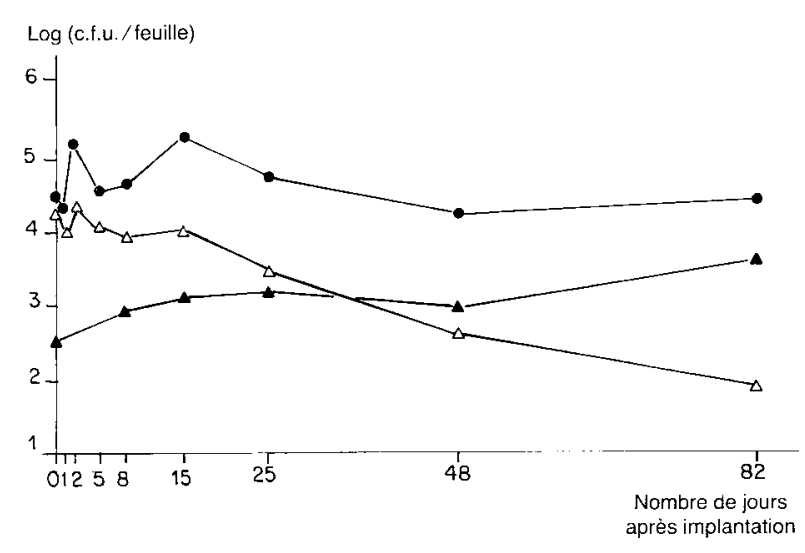

Figure 2

Evolution d'une population de Xanthomonas campestris $p v$. juglandis implantée sur feuilles de noisetier: $\triangle$ comptage après lavage, comptage après lavage et brovage, $\Delta$ flore naturelle des témoins.

Development of a Xanthomonas campestris $p v$. juglandis population settled on hazelnut leaves: $\triangle$ counting after washing, counting after washing and blending, $\boldsymbol{\Lambda}$ natural flora of control samples.

celles de la fig. 1, cependant la multiplication des bactéries est limitée puisque le nombre maximum de bactéries n'atteint que $10^{5}$ par feuille.

L'analyse de variance montre une différence significative à $1 \mathrm{p} .100$ entre les populations obtenues par lavage et les populations totales. Par contre, il n'y a pas de différences significatives des nombres de bactéries obtenus aux différentes dates d'analyse.

La comparaison des valeurs obtenues par les deux techniques d'analyse à chaque date par la p.p.d.s. ne montre qu'une différence presque significative à $5 \mathrm{p} .100$ à 47 jours et une différence significative à 82 jours.

L'utilisation du coefficient de Spearman montre une augmentation dans le temps de la différence entre les populations obtenues par lavage et les populations totales. Le nombre de bactéries saprophytes n'est que de 10 à 100 fois inférieures à celles de $X . c$. pv. juglandis.

Les fig. 3 et 4 montrent une dynamique d'implantation et de maintien comparable entre les deux bactéries saprophytes : $E$. herbicola et $P$. paucimobilis. Le nombre total de bactéries par feuille: les,

- est voisin de $10^{5}$ après la contamination des feuil-

- diminue pendant les deux premiers jours jusqu'à environ $10^{3}$,

- se stabilise ensuite entre $10^{3}-10^{4}$.

\section{Pour E. herbicola}

- l'analyse de variance montre une différence significative à 1 p. 100 entre le nombre de bactéries obtenues par lavage et la population totale ainsi qu'une différence significative à 5 p. 100 entre les effectifs bactériens en fonction des dates d'analyse,

- la comparaison deux à deux à chaque date des populations obtenues par lavage et des populations totales montre une différence significative à 5 p. 100 à 15,48 et 82 jours et presque significative à 25 jours, 


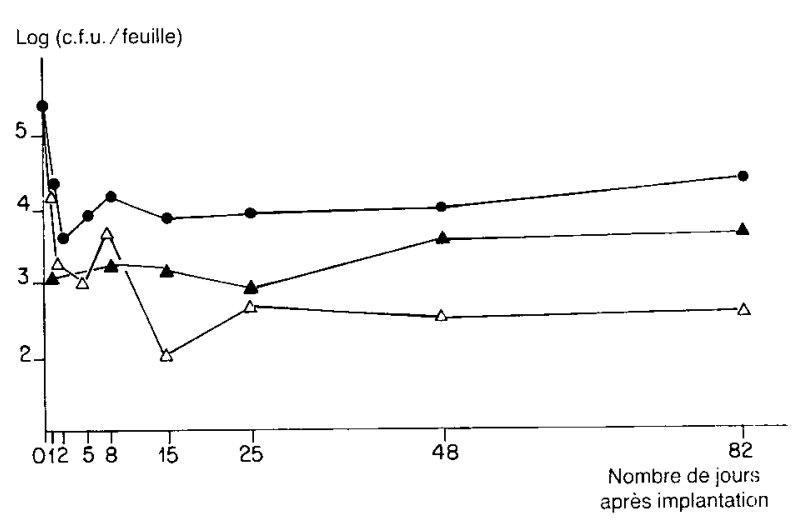

Figure 3

Evolution d'une population d'Erwinia herbicola implantée sur feuilles de noisetier: $\triangle$ comptage après lavage, - comptage après lavage et broyage, $\mathbf{A}$ flore naturelle des témoins.

Development of an Erwinia herbicola population settled on hazelnut leaves: $\triangle$ counting after washing, counting after washing and blending, $\mathbf{\Delta}$ natural flora of control samples.

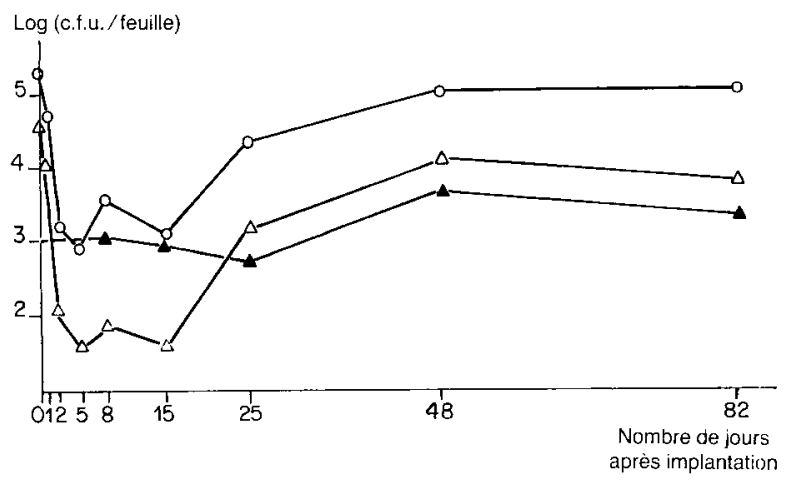

Figure 4

Evolution d'une population de Pseudomonas paucimobilis implantée sur feulles de noisetier : $\triangle$ comptage après lavage, 0 comptage après lavage et brovage, $\Delta$ flore naturelle des témoins.

Development of a Pseudomonas paucimobilis population setled on hazelnut leaves : $\triangle$ counting after washing, counting after washing and blending, $\boldsymbol{\Delta}$ natural flora of control samples.

- le calcul du coefficient de Spearman indique une augmentation significative de différences obtenues par les deux méthodes au cours du temps.

\section{Avec P. paucimobilis}

- l'analyse de variance des populations est significative à 1 p. 100 entre celles obtenues par les deux techniques et entre les différentes dates d'analyse,

- la comparaison des valeurs obtenues (par la p.p.d.s.) entre les techniques et par date d'analyse montre une différence presque significative à 1 p. 100 à 2,25 et 48 jours et significative à $5,8,15$ et 82 jours,

- le coefficient de Spearman ne montre pas une augmentation des différences entre les deux techniques au cours du temps.

Toutes les bactéries étudiées peuvent donc s'implanter et se maintenir sur les feuilles de Noisetier, cependant seule la bactérie pathogène homologue se multiplie très activement les 8 premiers jours et se maintient à un niveau très élevé de $10^{6}-10^{7}$ bactéries par feuille.

Il n'a pas été possible d'établir, dans nos conditions expérimentales, une corrélation entre les variations des populations bactériennes et les paramètres climatiques.

Pour les quatre bactéries, l'analyse de variance confirme des différences significatives entre les populations obtenues par lavage et broyage des feuilles. Cette différence moins marquée les 8 premiers jours de l'essai augmente ensuite ce qui suggère des modifications dans l'état et la localisation des bactéries sur la feuille; ce gradient a pu être mis en évidence à l'aide du coefficient de Spearman sauf pour $P$. paucimobilis.

Les comptages effectués sur les feuilles témoins donnent des nombres de bactéries saprophytes par feuille compris entre $10^{2}$ et $10^{3}$ ce qui est faible par rapport à ceux de $X . c$. pv. corylina mais non négligeable pour les trois autres espèces bactériennes étudiées; il faudra donc en tenir compte dans les observations au microscope électronique à balayage.

\section{B. Observations au microscope électronique à balayage}

Les bactéries saprophytes et les bactéries phytopathogènes se localisent sur la surface de la feuille en fonction du relief et de l'espèce bactérienne considérée.

\section{Anatomie de la feuille de noisetier (photos 1 et 2)}

L'observation macroscopique de la face supérieure de la feuille montre que les nervures créent dans le limbe des dépressions qui s'amenuisent le long de la nervure principale, de la base jusqu'à l'apex; le même phénomène est observé sur les nervures secondaires, tertiaires etc...

A la base de la feuille, la nervure principale forme néanmoins un léger relief au sein de la dépression; ce phénomène, observé aussi sur les autres nervures, a tendance à s'estomper, de leur base jusqu'à la périphérie de la feuille.

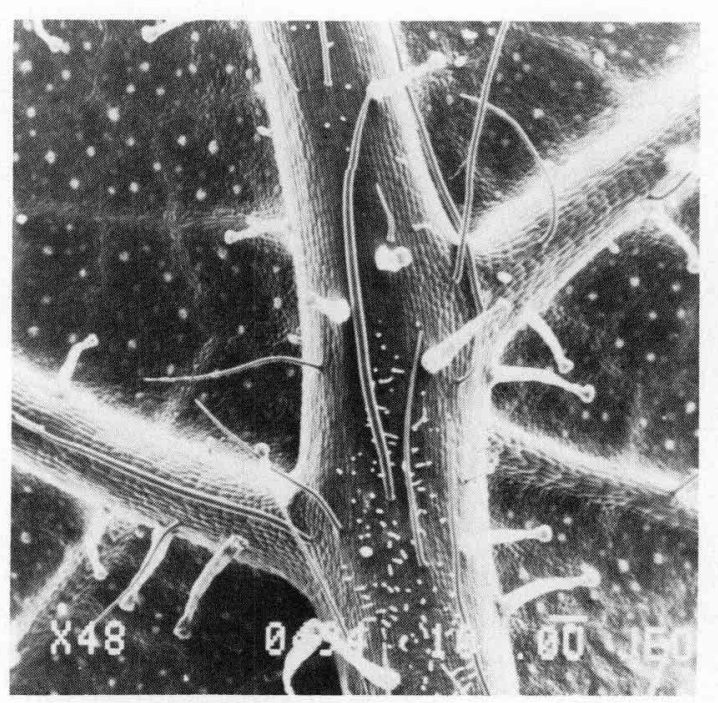

Photo 1

Face inferieure de feuille de noisetier montrant nervures principale et secondaire, limbe, stomates, trichomes et poils glandulaires.

Lower side of a hazelnut leaf showing main and secondary veins, lamina, stomata, trichomes and glandular trichomes. 


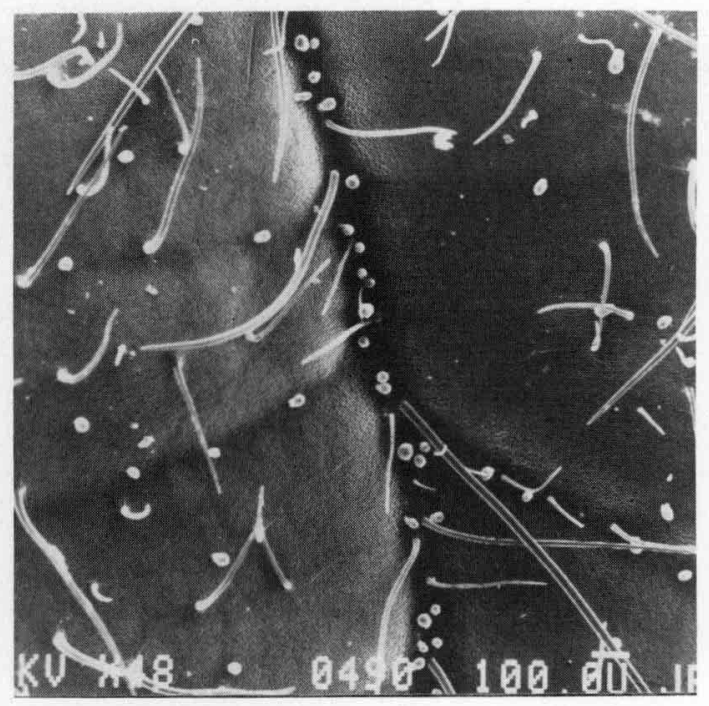

Photo 2

Face supérieure de feuille de noisetier montrant nervures principale of seconduire, limbe, trichomes et poils glandulaires.

Upper side of a hazelmut leaf showing main and secondary veins, lamina trichomes and glandular trichomes.

A la face inférieure, les nervures créent, au contraire, un relief important qui diminue progressivement de façon centrifuge de la base à l'apex et le long des nervures principales, secondaires, tertiaires, etc... à la périphérie de la feuille.

L'ensemble de la nervation au stade ultime se traduit dans le limbe par des figures polygonales de base qui semblent délimiter les taches foliaires de la bactériose du noisetier.

L'observation microscopique montre que la surface de la feuille comprend un épiderme constitué d'une assise continue de cellules de revêtement et stomatiques. Il existe également deux types de poils uni ou pluricellulaire : les trichomes qui ont un rôle de protection et les poils glandulaires qui ont un rôle de sécrétion.

Un examen approfondi permet d'apporter les précisions suivantes:

- la majorité des cellules de revêtement du limbe ont un aspect lisse en surface et ont une forme plus ou moins étoilée; les autres sont plissées en surface créant ainsi des dépressions,

- sur les nervures, les cellules de revêtement ont une forme allongée et sont plissées dans le sens longitudinal créant ainsi de très nombreuses dépressions,

- les poils sont implantés uniquement sur les nervures ; la pilosité semble identique sur les faces supérieure et inférieure de la feuille et elle décroît avec la grosseur des nervures. La pilosité des jeunes feuilles, du fait de leur taille, est plus dense que sur les feuilles âgées.

- les stomates ne sont localisés qu'à la face inféricure du limbe et ils induisent une augmentation du relief.

\section{Localisation des bactéries}

\section{a) X. c. $p v$. corylina}

Juste après l'inoculation, les bactéries sont réparties de façon hétérogène sur le limbe des faces supérieure et inférieure de la feuille (photo 3), de grandes plages sont

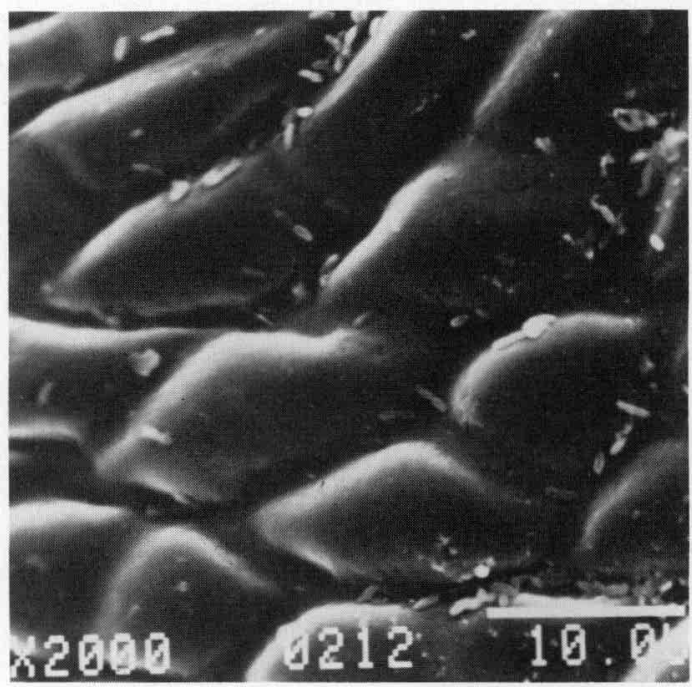

Photo?

X. c. pv. corylina

Bacteries isolés sur linhe de la face supérienre whe heure apress implantation.

Single hacteria on upper lamina one how after sethlement.

largement colonisées par les bactéries alors que d'autres en sont indemnes. Quelques bactéries isolées sont réparties au hasard à la surface des cellules épidermiques et des stomates, mais la majorité sont situées dans les dépressions existant au niveau des nervures mais aussi du limbe

Deux jours après la pollution des feuilles, les bactéries colonisent préférentiellement les dépressions des nervures et des zones proches du limbe; elles sont isolées, certaines en voie de division et leur nombre a augmenté de façon significative.

Au bout de cinq jours, l'aspect et la localisation des bactéries sont différents (photo 4 ):

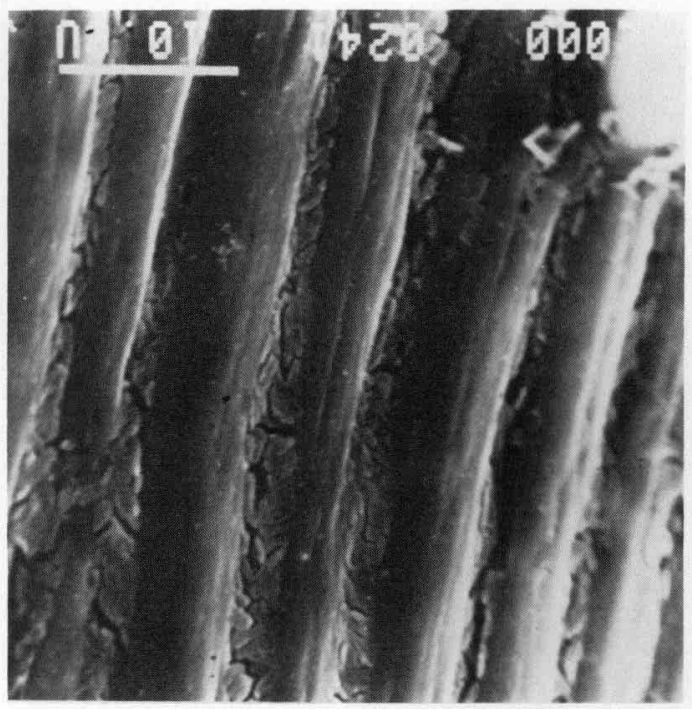

Photo 4

X. c. pve corylina

Amas de bactéries agghuinées dans les dépressions de la nervure principale (face infërieure) 5 jowrs après installation.

Agghtunated bacteria in depressions seen on the main vein (lower side) 5 days afier settlement. 
- elles ne sont désormais rencontrées que dans les dépressions même légères où elles forment des tapis contenus sur des plages plus ou moins étendues et très peu sont isolées.

les corps bactériens semblent agglutinés et enrobés dans un mucus qui se rétracte par endroits. Le phénomène s'accentuera au cours du temps.

Ces deux phénomènes peuvent permettre d'expliquer l'augmentation de la différence des populations obtenues par lavage et broyage des feuilles.

A partir de quinze jours, la fréquence d'observation des bactéries à la face supérieure diminue considérablement et celles-ci sont fortement agglutinées.

Dans cette expérience, les symptômes foliaires de la bactériose n'ont pu être observés. Les stomates n'ont pas été contaminés et n'ont pas rejeté des bactéries qui s'y seraient multipliées. Des bactéries ont cependant été vues sur et près des stomates mais la contamination n'aurait pu se faire qu'au stade précoce au moment où les bactéries sont isolées et dispersées de façon aléatoire; les paquets de bactéries agglutinées ne pourront donner des bactéries contaminatrices qu'après remise en suspension par l'eau de pluie ou de rosée.

Des colonies de levure sont observées à partir de 5 jours notamment à la base des trichomes.

Les feuilles témoins naturellement contaminées ont été observées aux mêmes dates, les faits suivants peuvent être notés :

- colonisation préférentielle de la face inférieure des feuilles avec une fréquence et un nombre faible de bactéries,

les bactéries sont toujours isolées, le plus souvent dans les dépressions des nervures ce que nous avons déjà observé sur des empreintes de feuilles entières dans des boîtes gélosées permettant la croissance des bactéries.

\section{b) Les trois autres bactéries}

Pendant les cinq premiers jours, aucune différence n'est observée avec X.c. pv. juglandis si ce n'est sa multiplication moins importante que l'on peut apprécier qualitativement lors des observations.

De 8 à 82 jours, les observations suivantes sont notées :

- comme pour X.c. pv. corvlina les cellules de X.c. pv. juglandis s'agglutinent dans un mucus qui se rétracte dans les dépressions (photo 5),

- les deux autres bactéries sont toujours sous forme isolées ou par petits paquets enfouis dans les dépressions des nervures et du limbe proche (photo 6).

En dehors des stomates, les sites de colonisation semblent donc être les mêmes pour toutes les bactéries.

\section{DISCUSSION}

Nous avons done montré que l'on peut installer sur noisetier en phase épiphylle des bactéries différentes: pathogène homologue, pathogène hétérologue et saprophyte. Cependant, seul $X$.c. pv. corylina a la possibilité de se multiplier très activement et se maintenir à un niveau très élevé $\left(10^{6}-10^{7}\right.$ c.f.u./feuuille) alors que pour

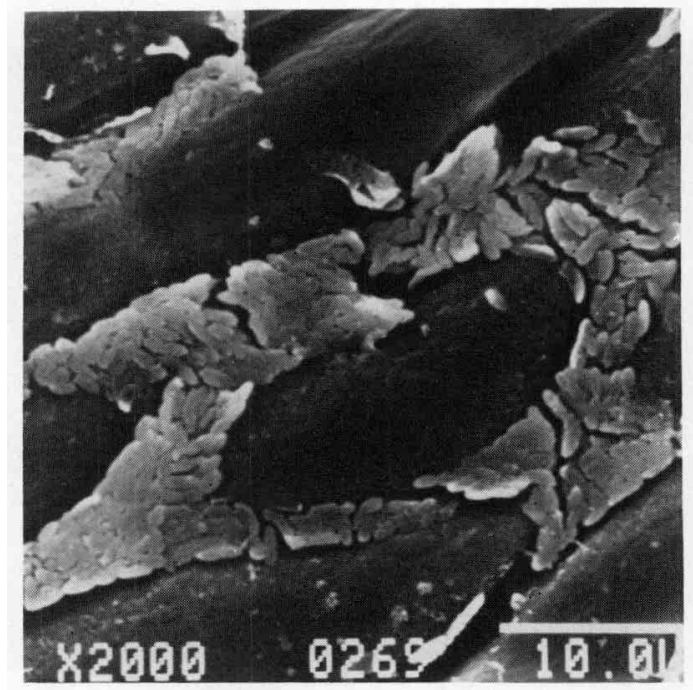

Photo 5

X.c. pv Juglandis

Amas de hacteries agghatinées dans les dépressions de la nervure principale (face supériewe) 8 jours après installation.

Agglutinated bacteria in depressions on the main vein (upper side) 8 dar's after seillement.

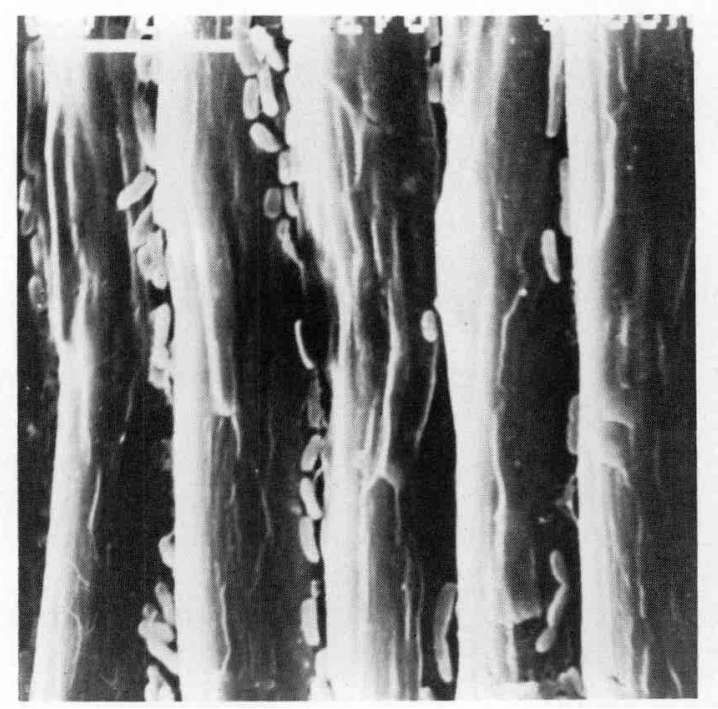

Pholo 6

Bactéries isolées P. paucimobilis dans lés dépressions de la nervare principale (face inferieure) 25 jours apres installation.

Single bacteria in the depressions seen on the main vein (lower side) 25 dars after solloment.

les autres. on ne dénombre que $10^{3}$ à $10^{5}$ c.f.u./feuille. Ces résultats sont en parfait accord avec ceux de YounG (1978) qui a montré que sur feuille de Prunus les espèces pathogènes se multipliaient abondamment alors qu' $E$. herbicola ne faisait que se maintenir à un niveau bas.

Une bactérie pathogène hétérologue semble se comporter de façnn assez similaire à une espèce saprophyte : il n'est pas rare en effet d'isoler les bactéries pathogènes à partir des feuilles de plantes herbacées, adventices par exemple, qui servent dans ce cas de réservoir d'inoculum.

L'évolution comparative d'une population homologue de $X$. c.pv. corylina par rapport à des populations hétérologues ou saprophytes, complétées par l'observation microscopique in situ suggère des relations privilégiées entre l'hôte et la bactérie homologue (qui seule se 
multiplie abondamment et se maintient à haut niveau). Une telle particularité repose peut-être sur la présence de certaines relations spécifiques.

L'augmentation des populations épiphylles de $X$. c. pv. corylina n'a pas, dans ce cas, été suivie d'un processus infectieux car les taches foliaires de bactériose ne sont pas apparues. Ceci peut être expliqué par l'existence, au moment de l'expérimentation d'une humidité insuffisante pour la pénétration de la bactérie dans les stomates. Par contre, RoOs \& HATTINGH (1983) ont observé une multiplication de $P$. syringae pv. mors-prunorum dans les stomates des feuilles de cerisier en l'absence complète de symptômes visibles. Nous pensons que pour $X . c$. pv. corylina la multiplication abondante des bactéries dans les chambres sousstomatiques avec émergence de bactéries à la surface de la feuille est à mettre en relation avec un processus infectieux au sens strict.

Les observations au microscope électronique à balayage permettent de mieux comprendre le phénomène de vie épiphylle :

- colonisation par les bactéries des dépressions le long des nervures ou des zones proches du limbe.

survie préférentielle à long terme des bactéries à la surface inférieure des feuilles,

- les Xanthomonas se maintiennent sous forme d'amas fortement agglutinés dans un mucus qui semble se rétracter; par contre, les bactéries saprophytes sont observées sous forme de cellules isolées ou de petits amas non agglutinés.

Nos observations peuvent être mises en relation avec les travaux de TAKAHASHI \& DOKE (1985) qui ont purifié, à partir de feuille de Citrus, une agglutinine glycoprotéique qui agit sur l'exopolysaccharide de $X$. campestris pv. citri bactérie pathogène homologue et de $X$. c. pv. campestris, bactérie pathogène hétérologue. Celà pourrait expliquer l'aspect des cellules de $X$. c. pv. corylina et $X . c$. pv. juglandis à la surface des feuilles de noisetier. On peut aussi penser que le mucus naturellement produit en culture par les Xanthomonas est produit, même en plus faible quantité, sur les feuilles, se déssèche et se craquèle sous l'effet de la température. Sous des conditions climatiques contrôlées, il faudrait vérifier la réhydratation de ce mucus et la redispersion des bactéries sur le limbe.

Toutes ces observations permettent de mieux comprendre comment et pourquoi $X$. c. pv. corvlina se maintient pendant toute la période végétative du noisetier (GARDAN \& DEVAUX, 1984) alors que pour d'autres bactéries comme $P$. syringae pv. persicae, les populations tombent à niveau très bas sur les feuilles de pêcher pendant les périodes très sèches (GARDAN et al. 1972).

La localisation préférentielle des bactéries dans les dépressions et leur présence en grand nombre à la face inférieure des feuilles sont probablement dues à des conditions microclimatiques très favorables à la survie et à la multiplication des bactéries dans ces sites. La quasi-disparition des bactéries de la surface supérieure des feuilles pourrait s'expliquer en outre par l'action des rayonnements ultraviolets solaires et par une humidité relative plus faible.

Les différences importantes de population obtenues par les techniques de broyage et de lavage peuvent en partie s'expliquer par l'incrustation, dans les sites protégés que sont les dépressions, sous forme d'amas agglutinés. Il ne faut pas exclure toutefois la possibilité de présence de bactéries dans les chambres sousstomatiques qui ne seraient détectées que par la technique du broyage et peut-être aussi l'existence de phénomène d'association des bactéries avec la surface du limbe. LEBEN \& WhITMOYER (1979) ont d'ailleurs étudié l'association de $P$. s. pv. lachrymans avec des feuilles de concombre.

Pour P.s. pv. persicae, LUISETTI \& GAIGNARD (1984) émettent l'hypothèse qu'une bonne partie des bactéries épiphylles viennent de microlésions sur la base de différences de comptage des bactéries par empreintes, lavages et broyages. Ces microlésions qui n'ont pas été localisées, seraient des sites d'infection atténuée. Nous avons observé de larges îlots de colonisation des nervures et du limbe, qui dans certains cas ressemblent à des micro-colonies; ces sites sont-ils spécifiques ou répartis de façon aléatoire?

\section{Recule 7 mars 1988 Accepte le 11 septembre 1988}

\section{REMERCIEMENTS}

Nous remercions R. FILMON UER des Sciences Médicales et Pharmaceutiques - Université d'Angers pour son aide et ses conseils lors des obscrvations en microscopie électronique à balayage.

\section{RÉFÉRENCES BIBLIOGRAPHIQUES}

Bashan Y., Sharon E., Okon Y., Henis Y., 1981. Scanning electron microscopy and light microscopy of infection and symplom development in tomato leaves infected with Pseudomonas tomato. Phisiol. Plant Pathol., 19, 139-144.

Crosse J. E., 1963. Bacterial canker of stone fruits. V. A comparison of leaf surface populations of Pseudomonas morsprunorum in autumn on two cherry varieties. Am. Appl. Biol. 52, 96-104.

Daniel J. F., Boher B., 1978. Ecology of cassava bacterial blight: epiphytic survival of Xanthomonas manihotis on aerial parts of the cassava plant. Proc 4 th Int. Conf. Plant Path. Bact., Angers 11. $763-771$

Dhanvantari B. N., 1973. Population dynamics of Xanthomonas prumi on peach bud and leaf surface and its relation to other microflora. Ahstr. Papers 2nd Int. Cong. of Plant Path., Minneapolis.

Gardan L., Devaux M., 1984. Bacterial blight of hazelnut caused by
Xanthomonas corllina. Comegno internasionale sul nocciwolo. Avellino, Italia, 1983, 443-448.

Gardan L., Prunier J. P., Luisetti J., 1972. Etudes sur les bactérioses des arbres fruitiers. IV. Recherche et étude des variations de Pseudemonas morsprunorum f. sp. persicae à la surface des feuilles de Pêcher Ann. Phytopathol., 4, (3) 229-244.

Gitaitis R. D., Samuelson D. A., Strandberg J. O., 1981. Scanning electron microscopy of the ingress and establishment of Pseudomonos alboprecipitans in sweet corn lcaves. Phytopathology, 71, (2). 171-175. Leben C., 1965. Epiphytic microorganisms in relation to plant disease. Annu. Rev. Phytopathol., 3, 209-230

Leben C., 1969. Colonization of soybean buds by bacteria : observation with the scanning electron microscope. Can. J. Microbiol., 15 , 319-320

Leben C., 1974. Survival of plant pathogenic bacteria. Spec. Circ. 10\%) Ohio Agric. Res. Cent. Wooster, OH. p. 21 
Leben C., Daft G. C., 1964. Characteristics of bacteria isolated from leaves of cucumber seedlings. Can. J. Microbiol., 10,919-923.

Leben C., Whitmoyer R. E., 1979. Adherence of bacteria to leaves. Can. J. Microbiol., 25, 896-901.

Luisetti J., Gaignard J. L., 1984. Variations in the distributicn of Pseudomonas persicae epiphytic populations. Proc. 2nd working group on Pseudomonas syringae pathorars. Sounion (Greece), 24-28 April 1984, 17-18.

Mew T. W., Mew I. C., Huang J. S., 1984. Scanning electron microscopy of virulent and avirulent strains of Xanthomonas campestris pv. oryzae on rice leaves. Phytopathology, 74, $\mathrm{n}^{\circ} 6,635-641$.

Miles N. G., Daines R. H., Rue J. W., 1977. Presymptomatic egress of
Xanthomonas pruni from infected peach leaves. Phytoparhology, 67, 895-897.

Roos I. M. M., Hattingh M. J., 1983. Scanning electron microscopy of Pseudomonas syringac $\mathrm{pv}$. morsprtanom on sweet cherry leaves. Phytopathol. Z., 108, N*1, 18-25

Schneider R.W., Grogan R. G., 1977. Tomato leaf trichomes, a habitat for resident populations of Pseudomonas tomato. Phytopatho$\log 1.67,898-902$.

Takahashi T., Doke N., 1985. Purification and partial characterization of an agglutinin in citrus leaves against extracellular polysaccharides of Xanthomonas campestris pv. citri. Physiol. Plant Pathol., 27, 1-13. Young J. M., 1978. Survival of bacteria on Prthms leaves. Proc. 4th Iit. Conf. Plant Path. Bact., Angers, 11, 779-786. 